\title{
Comparative analysis of regional outcomes and adverse events after continuous-flow left ventricular assist device implantation: An IMACS analysis
}

\author{
Kiran K. Mirza, MD, ${ }^{\mathrm{a}}$ Rongbing Xie, $\mathrm{DrPH}, \mathrm{MPH},{ }^{\mathrm{b}}$ Jennifer Cowger, MD, MS, ${ }^{\mathrm{c}}$ \\ James K. Kirklin, MD, ${ }^{b}$ Bart Meyns, MD, PhD, ${ }^{d}$ \\ Finn Gustafsson, MD, PhD, DMSc, ${ }^{\text {a, e }}$ Steven M. Shaw, MBChB, $\mathrm{PhD}^{f}{ }^{f}$ and \\ Daniel J. Goldstein, MD ${ }^{g}$
}

From the ${ }^{a}$ Department of Cardiology, Rigshospitalet, Copenhagen, Denmark; ${ }^{b}$ Department of Surgery, University of Alabama, Birmingham, Alabama; ${ }^{c}$ Division of Cardiovascular Medicine, Henry Ford Hospital, Detroit, Michigan; ${ }^{d}$ Department of Clinical Cardiac Surgery, Katholieke Universiteit Leuven, Leuven, Belgium; ${ }^{e}$ Department of Clinical Medicine, University of Copenhagen, Copenhagen, Denmark; ${ }^{f}$ Manchester University NHS Foundation Trust, Manchester, United Kingdom; and the ${ }^{g}$ Department of Cardiothoracic Surgery, Montefiore Medical Center, Bronx, New York.

\section{KEYWORDS:}

left ventricular assist device; continuous flow; Americas; Asia-Pacific; Europe; outcome

\begin{abstract}
INTRODUCTION: Regional outcomes after implantation of continuous-flow left ventricular assist devices (LVADs) have not been described. We examined differences in patient selection, survival, and adverse events across 3 geographic regions of the world: the Americas, Asia-Pacific, and Europe.

METHODS: Using data from The International Society for Heart and Lung Transplantation Mechanically Assisted Circulatory Support registry, all adult patients implanted with a continuous-flow LVADs were included in this International Society for Heart and Lung Transplantation Mechanically Assisted Circulatory Support analysis $(n=15,560)$, of whom, 9,988 $(64 \%)$ received axial-flow devices and $5,572(36 \%)$ received centrifugal-flow devices.

RESULTS: There were significant interregional differences in the rate of implantation of patients aged $>70$ years (Americas: 14\%, Asia-Pacific: $1 \%$, Europe: $5 \% ; p<0.0001$ ), morbidly obese (Americas: 5\%, Asia-Pacific: $1 \%$, Europe: $1 \% ; p<0.0001$ ), male (Americas: $79 \%$, Asia-Pacific: $77 \%$, Europe: $85 \% ; p<0.0001$ ), and implanted as destination therapy (Americas: $48 \%$, Asia-Pacific: $4 \%$, Europe: $22 \% ; p<0.0001$ ). The rates of centrifugal pump usage varied by region (Americas: $30 \%$, Asia-Pacific: $34 \%$, Eu: $74 \% ; p<0.0001$ ). Survival rates varied by region and the type of pump flow, with survival at 12 and 48 months (axial flow vs centrifugal flow) being $82 \%$ vs $82 \%$ and $52 \%$ vs 53 in Americas; $92 \%$ vs $86 \%$ and $83 \%$ vs $74 \%$ in Asia-Pacific; and $80 \%$ vs $75 \%$ and $69 \%$ vs $53 \%$ in Europe, respectively (regional survival $p<0.0001$ ).

CONCLUSION: There are marked global differences in LVAD recipient characteristics, device utilization, and post-operative care. These heterogeneities along with differences in patient management and transplantation rates may impact long-term survival. Regional differences in adverse event incidence warrant further investigation. J Heart Lung Transplant 000;000:1-11

(C) 2020 International Society for Heart and Lung Transplantation. All rights reserved.
\end{abstract}

Reprint requests: Kiran K. Mirza, Department of Cardiology, Copenhagen University Hospital, Rigshospitalet, Blegdamsvej 9, DK-2100
Copenhagen, Denmark. Telephone: +45 35451442. Fax: +4535453870 E-mail address: RNZ440@alumni.ku.dk 
Continuous-flow left ventricular assist devices (CFLVADs) have become the preferred mode of mechanical circulatory support used in patients with advanced chronic heart failure worldwide. $^{1-5}$ Reports from America, ${ }^{2,6,7}$ Asia, ${ }^{8,9}$ and Europe $^{3,5}$ describe an improved survival with reduced incidence of adverse events (AEs) especially when compared with earlier technologies. ${ }^{1,10,11}$ However, studies examining differences in indication, survival, and AEs among the regions of the world are lacking.

Current data suggest that regional disparities exist in waiting times for heart transplantation in America, ${ }^{12,13}$ Europe, ${ }^{14,15}$ and Asia. ${ }^{16,17}$ Such disparities, among others, could lead to differences in device utilization and indications for implantation among the regions. As geographic variation in indications, regulatory environments, and/or management protocols could lead to different outcomes, identification of these regional differences offers an opportunity to optimize global use of LVADs, generate new knowledge that may be shared across regions, and establish best practices. Furthermore, understanding regional differences is vital when planning and interpreting multicenter international trials in the field.

For these reasons, we examined worldwide differences in indications and outcomes after implantation of CFLVADs on the basis of the data from The International Society for Heart and Lung Transplantation Mechanically Assisted Circulatory Support (IMACS) registry.

\section{Methods}

\section{Data source and study population}

This study relied on data from the adult recipients of CFLVADs in the IMACS registry (Figure 1), which was arbitrarily divided into 3 geographic regions of the world: the Americas, Europe, and Asia-Pacific (refer to Supplementary Figure S1 available online at www.jhltonline.org).

In brief, data were entered in the registry from 2 general sources: multicenter collectives (Interagency Registry for Mechanically

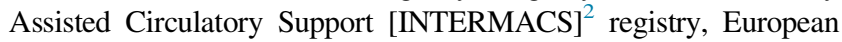

Registry for Patients with Mechanical Circulatory Support ${ }^{18}$ registry, United Kingdom registry, and the Japanese Registry for Mechanically Assisted Circulatory Support ${ }^{8}$ registry) and individual hospitals ( $n=24$, IMACS website). ${ }^{1}$ Submission of data is mandatory for centers participating in INTERMACS, United Kingdom, and Japanese Registry for Mechanically Assisted Circulatory Support registries. The INTERMACS database excluded all data from patients with experimental devices. For European Registry for Patients with Mechanical Circulatory Support, data entering is not mandatory; however, participating hospitals do commit to enter consecutive data on implanted participants by 6 weeks of device implantation (if expected support duration is $\geq 6$ months). ${ }^{5}$ Implant data included in this report range from January 1, 2013 to November 1, 2017, encompassing 16,754 patients (age $\geq 18$ years) implanted with a CFLVAD in the 31 different countries reporting to IMACS. ${ }^{1}$

\section{Definitions of variables and outcomes}

The primary outcomes were all-cause mortality and AEs by region (overall and by device type [axial vs centrifugal]) with data censored at transplantation or device removal. AEs discussed in this report (right heart failure, major bleeding, cardiac arrhythmia, hemolysis, major infection, device malfunction, hepatic dysfunction, renal dysfunction, neurologic dysfunction) follow established INTERMACS definitions.

\section{Statistical methods}

Continuous normally distributed variables were expressed as mean $\pm \mathrm{SD}$ and as median and interquartile range if non-parametric. Categorical variables were described with frequencies and percentages. Survival was estimated using the Kaplan-Meier method and the log-rank test.

Competing outcomes depictions were created displaying 4 mutually exclusive outcomes: alive (with device still in place), death (before transplant), transplant, and explant (device removal, see Supplementary Table S1 online). Statistical significance was defined as a 2-tailed $p$-value below 5\%. The findings in this report were reviewed and approved by the IMACS Research Committee.

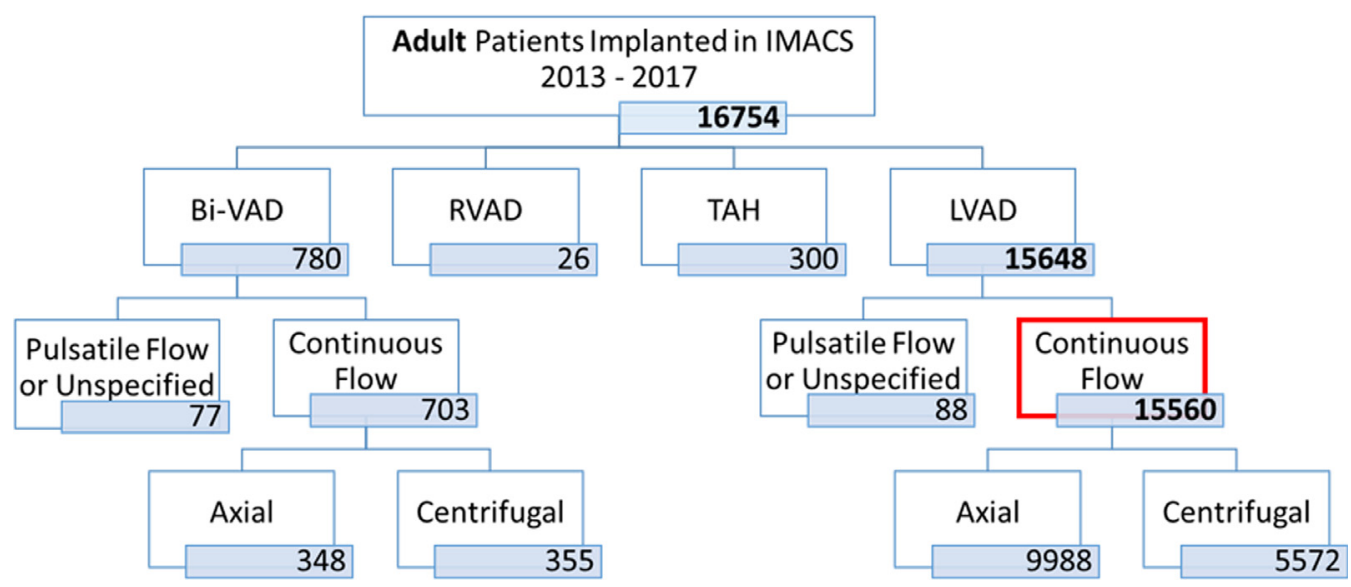

Figure 1 Patient disposition. All adult recipients of continuous-flow LVAD ( $n=15,560$, red marking) were included in this study. Of these patients, 9,988 received an Ax device and 5,572 received a Centr device. Ax, axial flow; BiVAD, biventricular assist device; Centr, centrifugal flow; IMACS, The International Society for Heart and Lung Transplantation Mechanically Assisted Circulatory Support registry; LVAD, left ventricular assist device; RVAD, right ventricular assist device; TAH, total artificial heart. 


\section{Results}

Of the 16,754 patients receiving mechanical circulatory support within the study dates, 780 patients received a biventricular assist device, 26 received a right ventricular assist device, and 300 received a total artificial heart and were all excluded. The remaining 15,648 patients received an LVAD of pulsatile $(n=88)$ or continuous $(n=15,560)$ flow type. Only the latter patients are the focus of this report, and among these, 9,988 (64\%) were implanted with an axial-flow (Ax) device and 5,572 (36\%) were implanted with a centrifugal-flow (Centr) device (Figure 1). The patient contribution of each region is shown in Table 1.

\section{Patient demographics and device types}

The largest proportion of patients were implanted in the Americas (82\%), whereas Asia-Pacific was the region with

Table 1 Baseline Characteristics in Each Region

\begin{tabular}{|c|c|c|c|c|c|}
\hline Characteristics & $\begin{array}{l}\text { Americas, } \\
n=12,942\end{array}$ & $\begin{array}{l}\text { Asia-Pacific, } \\
n=742\end{array}$ & $\begin{array}{l}\text { Europe, } \\
n=1,876\end{array}$ & $\begin{array}{l}\text { Global, } \\
n=15,560\end{array}$ & $p$-value \\
\hline Female, $\%$ & 21 & 23 & 15 & 21 & $<0.0001$ \\
\hline Age at implantation, years & & & & & $<0.0001$ \\
\hline $18-29$ & 5 & 11 & 7 & 5 & \\
\hline $30-49$ & 21 & 41 & 25 & 23 & \\
\hline $50-60$ & 60 & 47 & 63 & 60 & \\
\hline$>70$ & 14 & 1 & 5 & 12 & \\
\hline BMI, \% & & & & & $<0.0001$ \\
\hline Underweight & 4 & 21 & 3 & 5 & \\
\hline Non-obese & 59 & 75 & 76 & 62 & \\
\hline Obese & 32 & 4 & 20 & 29 & \\
\hline Morbidly obese & 5 & 1 & 1 & 5 & \\
\hline Chronic renal disease, $\%$ & 22 & 17 & 11 & 21 & $<0.0001$ \\
\hline Chronic pulmonary disease, $\%$ & 9 & 2 & 3 & 8 & $<0.0001$ \\
\hline History of stroke, $\%$ & 4 & 4 & 9 & 4 & $<0.0001$ \\
\hline Severe diabetes, \% & 10 & 15 & 12 & 10 & $<0.0001$ \\
\hline \multicolumn{6}{|l|}{ Tricuspid regurgitation, \% (moderate/severe) } \\
\hline Yes & 42 & 41 & 39 & 42 & 0.08 \\
\hline Days of hospitalization & $25 \pm 32$ & $82 \pm 70$ & $43 \pm 42$ & $29 \pm 37$ & $<0.0001$ \\
\hline ICU days & $11 \pm 14$ & $13 \pm 16$ & $17 \pm 22$ & $12 \pm 15$ & $<0.0001$ \\
\hline$A x, \%$ & 70 & 66 & 26 & 64 & $<0.0001$ \\
\hline Centr, \% & 30 & 34 & 74 & 36 & $<0.0001$ \\
\hline Device strategy, \% & & & & & $<0.0001$ \\
\hline Listed for transplant & 26 & 56 & 38 & 29 & \\
\hline Candidacy to transplant & 26 & 40 & 34 & 28 & \\
\hline Destination therapy & 48 & 4 & 22 & 42 & \\
\hline Other & 1 & 0 & 6 & 1 & \\
\hline INTERMACS profiles, \% & & & & & $<0.0001$ \\
\hline 1 & 16 & 11 & 12 & 15 & \\
\hline 2 & 34 & 40 & 30 & 34 & \\
\hline 3 & 35 & 41 & 30 & 35 & \\
\hline 4 & 12 & 6 & 20 & 13 & \\
\hline 5 & 2 & 1 & 4 & 2 & \\
\hline 6 & 1 & 0 & 2 & 1 & \\
\hline 7 & 0 & 0 & 1 & 0 & \\
\hline IABP, yes \% & 31 & 27 & 10 & 29 & $<0.0001$ \\
\hline ECMO, yes \% & 5 & 6 & 9 & 6 & $<0.0001$ \\
\hline Any concomitant surgery, $n(\%)$ & $5,357(41)$ & $344(47)$ & $547(29)$ & $6,248(40)$ & $<0.0001$ \\
\hline Aortic valve repair/replacement, $n(\%)$ & $656(5)$ & $44(6)$ & $83(4)$ & $783(5)$ & 0.24 \\
\hline Mitral valve repair/replacement, $n(\%)$ & $451(3)$ & $44(6)$ & $27(1)$ & $522(3)$ & $<0.0001$ \\
\hline Tricuspid valve repair/replacement, $n(\%)$ & $166(1)$ & $206(28)$ & $140(7)$ & $512(3)$ & $<0.0001$ \\
\hline CABG, $n(\%)$ & $183(1)$ & $6(1)$ & $22(1)$ & $211(1)$ & 0.30 \\
\hline PF0 closure, $n(\%)$ & $689(5)$ & $34(5)$ & $60(3)$ & $783(5)$ & $<0.001$ \\
\hline Other concomitant surgery, $n(\%)$ & $3,443(27)$ & $65(9)$ & $260(14)$ & $3,768(24)$ & $<0.0001$ \\
\hline
\end{tabular}

Abbreviations: Ax, axial flow; BMI, body mass index; CABG, coronary artery bypass grafting; Centr, centrifugal flow; ECM0, extracorporeal membrane oxygenation; IABP, intra-aortic balloon pump; ICU, intensive care unit; INTERMACS, Interagency Registry for Mechanically Assisted Circulatory Support; PF0, patent foramen ovale.

Continuous values are noted as mean $\pm \mathrm{SD}$, and proportions are noted as percentage (\%). Data were compared using chi-square test for categorical variables and $t$-test for continuous variables. It should be noted that procedures are not mutually exclusive. 
the fewest implantations (Table 1). The Americas implanted the highest frequency of patients aged $\geq 70$ years. Globally and regionally, most recipients were male, and the proportion of female recipients was lowest in Europe (15\%, $p<0.0001$ ). Body mass index (BMI) clearly differed between regions as $21 \%$ of those implanted in the Americas were morbidly obese. Conversely, $21 \%$ of those implanted in Asia-Pacific were underweight.

(a)

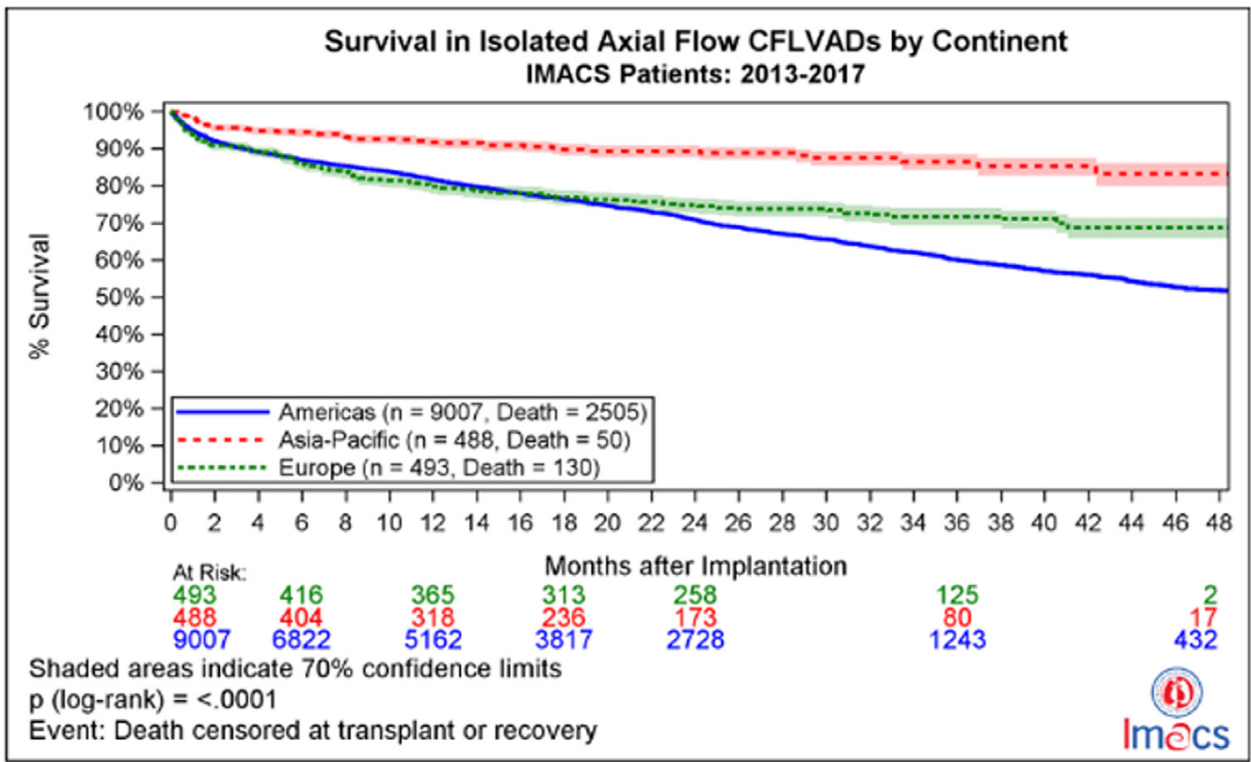

\begin{tabular}{|l|l|l|l|}
\hline Months after Implant & Americas & Asia-Pacific & Europe \\
\hline 1 & $94.8 \%$ & $98.4 \%$ & $93.9 \%$ \\
\hline 12 & $81.6 \%$ & $91.7 \%$ & $79.8 \%$ \\
\hline 24 & $70.8 \%$ & $89.3 \%$ & $74.7 \%$ \\
\hline 48 & $51.9 \%$ & $83.2 \%$ & $68.7 \%$ \\
\hline
\end{tabular}

(b)

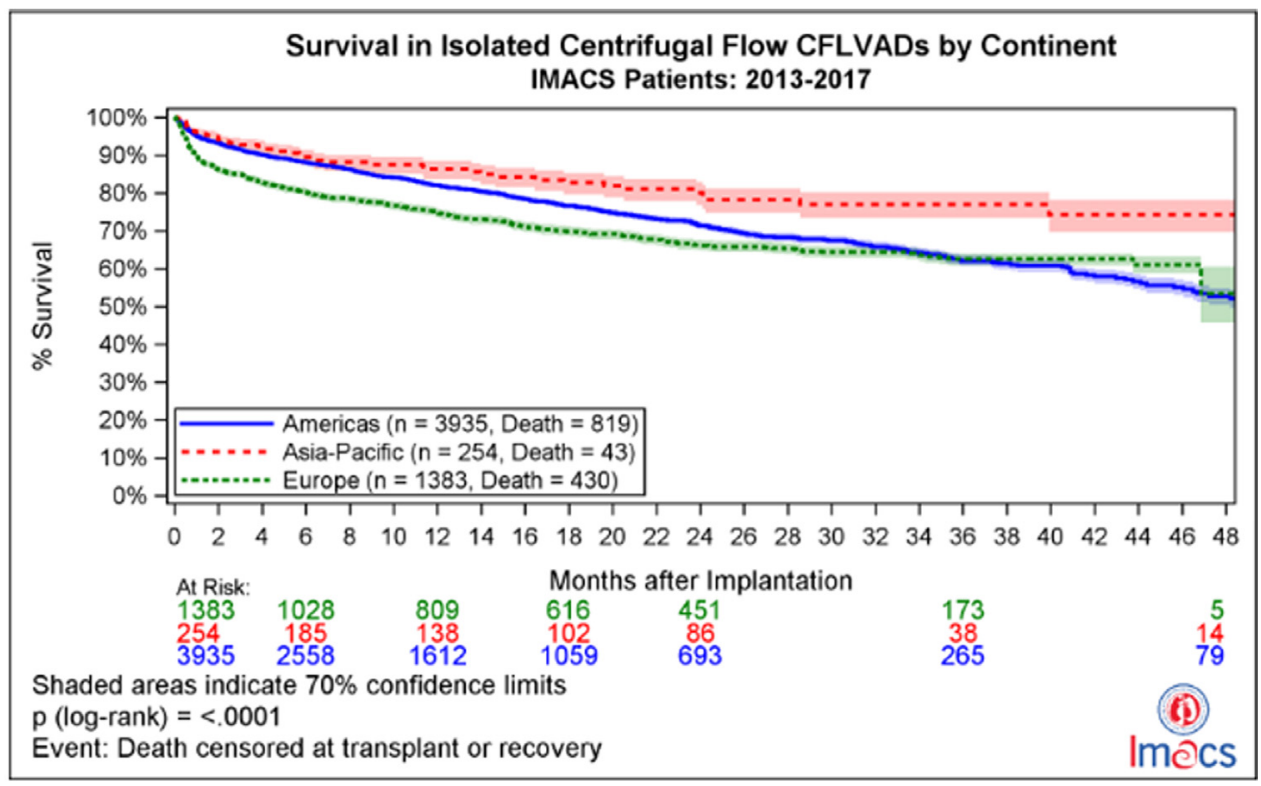

\begin{tabular}{|l|l|l|l|}
\hline Months after Implant & Americas & Asia-Pacific & Europe \\
\hline 1 & $95.1 \%$ & $96.0 \%$ & $89.9 \%$ \\
\hline 12 & $82.0 \%$ & $86.3 \%$ & $74.7 \%$ \\
\hline 24 & $71.4 \%$ & $80.0 \%$ & $66.3 \%$ \\
\hline 48 & $52.8 \%$ & $74.2 \%$ & $53.4 \%$ \\
\hline
\end{tabular}

Figure 2 Competing outcomes in the IMACS registry according to pump flow. (a) Ax pumps. (b) Centr pumps. Ax, axial flow; Centr, centrifugal flow; CFLVAD, continuous-flow left ventricular assist device; IMACS, The International Society for Heart and Lung Transplantation Mechanically Assisted Circulatory Support registry. 
Device indication varied across the globe with AsiaPacific being the region with the most patients listed for transplant at baseline. The region with the highest proportion of destination therapy was clearly the Americas (Americas $48 \%$ vs Asia-Pacific $4 \%$ vs Europe $22 \%, p<0.0001$ ).

Globally, Ax devices were most commonly implanted during the time of registration in the IMACS database (64\%), but in Europe, Centr devices were most commonly implanted (74\% of all implants).

Days of hospitalization was lowest in Americas (mean: 25 days) and highest in Asia-Pacific (mean: 82 days), whereas days in the intensive care unit was highest in Europe (mean: 17 days) and lowest in the Americas (mean: 11 days). Interestingly, although INTERMACS 2 and 3 profiles predominate on a worldwide basis, $27 \%$ of patients in Europe were not inotrope-dependent (Profile $\geq 4$ ) compared with $15 \%$ in the Americas and 7\% in Asia-Pacific. The Asia-Pacific region demonstrated a very aggressive attitude toward the repair of the tricuspid valve $(28 \%)$ when compared with other regions despite the fact that the incidence of moderate or severe tricuspid regurgitation was very similar between the regions. The regional incidence of tricuspid regurgitation and tricuspid valve repair are shown in Supplementary Table S2 online.

\section{Survival}

Global survival at 1, 12, 24, and 48 months was $94.5 \%$, $81.4 \%, 71.6 \%$, and $54.7 \%$, respectively. At the same time points, global survival rates stratified by Ax vs Centr device flow were $94.9 \%$ vs $93.9 \%, 82.0 \%$ vs $80.4 \%, 72.0 \%$ vs $70.7 \%$, and $54.2 \%$ vs $56.3 \%$, respectively. Regionally, survival rates at 1, 12, 24, and 48 months were highest in AsiaPacific for both the flow types; moreover, extended survival ( $>3$ years) was more often seen in Asia-Pacific ( $p<$ 0.0001 , Figure 2). Further total CFLVAD survival is shown in Supplementary Figure S2 online.

The likelihood of transplantation within the first 6 months after implantation among patients actively listed for heart transplantation at the time of implantation are shown in Supplementary Figures S3-S5 online, and competing outcomes worldwide are shown in Figure 3. Further investigation of age, gender, device therapy, and INTERMACS profiles as factors driving the survival differences in the 3 regions revealed that all these factors were significant predictors of poorer survival (Supplementary Table S3 online). In fact, Multiphase hazard analysis revealed that the risk of death in the first 3 months after implantation was highest in the European region (hazard ratio: 2.7, 95\% CI: 2.26, 3.22, $p<0.01$ ), and the risk of death $>3$ months after implantation was highest in the region of the Americas (hazard ratio: $1.55,95 \%$ CI: $1.35,1.78, p<0.01)$. Multivariate analyses predicting survival identified several significant regional variations that may contribute to the regional differences in survival in the early post-implant phase (female gender, age per 10-year increase, ischemic heart failure etiology, creatinine [per 1-mg/dl increase], white blood cell count [per 100 unit $/ \mu$ l increase], and device indication [i.e., destination therapy]). Factors of importance for the early-phase survival only were BMI (per 5-unit increase) and, not surprisingly, moderate or severe tricuspid regurgitation, pulmonary artery diastolic pressure (per 10-mm $\mathrm{Hg}$ increase), central venous pressure (per 10-mm $\mathrm{Hg}$ increase), total bilirubin (per 1-mg/dl increase), sodium (per 10-mEq/ liter increase), aspartate aminotransferase (per $50 \mu /$ liter increase), pre-implant extracorporeal membrane oxygenation, pre-implant ventilator/intubation, and pre-implant dialysis. For late-phase survival, blood type $\mathrm{A}, \mathrm{B}$, or $\mathrm{AB}$ vs $\mathrm{O}$, peripheral vascular disease, blood urea nitrogen (per 25$\mathrm{mg} / \mathrm{dl}$ increase), and, not surprisingly, the use of preimplant intra-aortic balloon pump were found as significant factors among regions. Interestingly, left ventricle Centr was also found as a factor that differed significantly among regions. All $p$-values $<0.01$

\section{AEs}

The rates of major events (the 5 most common stratified by flow type) per 100 patient-month are listed in Table 2. In the early phase ( $<3$ months), gastrointestinal bleed (GIB) was the most common $\mathrm{AE}$ in the Americas and globally, regardless of the flow type. In the early phase, the most common $\mathrm{AE}$ in Asia-Pacific was device-specific infection for Ax devices, whereas GIB was the most common AE in the Centr devices. In Europe, pump thrombosis was the most common $\mathrm{AE}$ for both the flow types. All AE risks declined after the first 3 months after implantation. At 6 months, freedom from first pump thrombosis (Figure 4a), cerebrovascular attack (Figure 4b), device-specific infection (Figure 4c), and GIB (Figure 4d) were lower in Europe, although freedom from pump thrombosis $>12$ months was lowest in Asia-Pacific (Figure 4a).

\section{Cause of death}

The 3 most common causes of death worldwide were similar among regions (neurologic dysfunction, multiorgan failure, and major infections), although clear differences were seen between the types of devices (Table 3). Neurologic dysfunction was most often seen in both flow types in the Americas and Asia-Pacific, whereas this was the second most common cause of death in European patients with Ax devices. The most common cause of death in Europe was multisystem organ failure in patients with Ax devices and infection in those with Centr devices. Although infection was observed as the second most common cause of death in Asia-Pacific, it is noteworthy that this was found as the fourth most common cause of death in the Americas. Furthermore, pump thrombosis was a rare cause of death in any region regardless of the flow type.

\section{Discussion}

The main findings of the present IMACS analysis, the first to compare regional differences in outcomes, are that the patients undergoing durable LVAD implantation in the 
(a)

Competing Outcomes-Axial: Overall

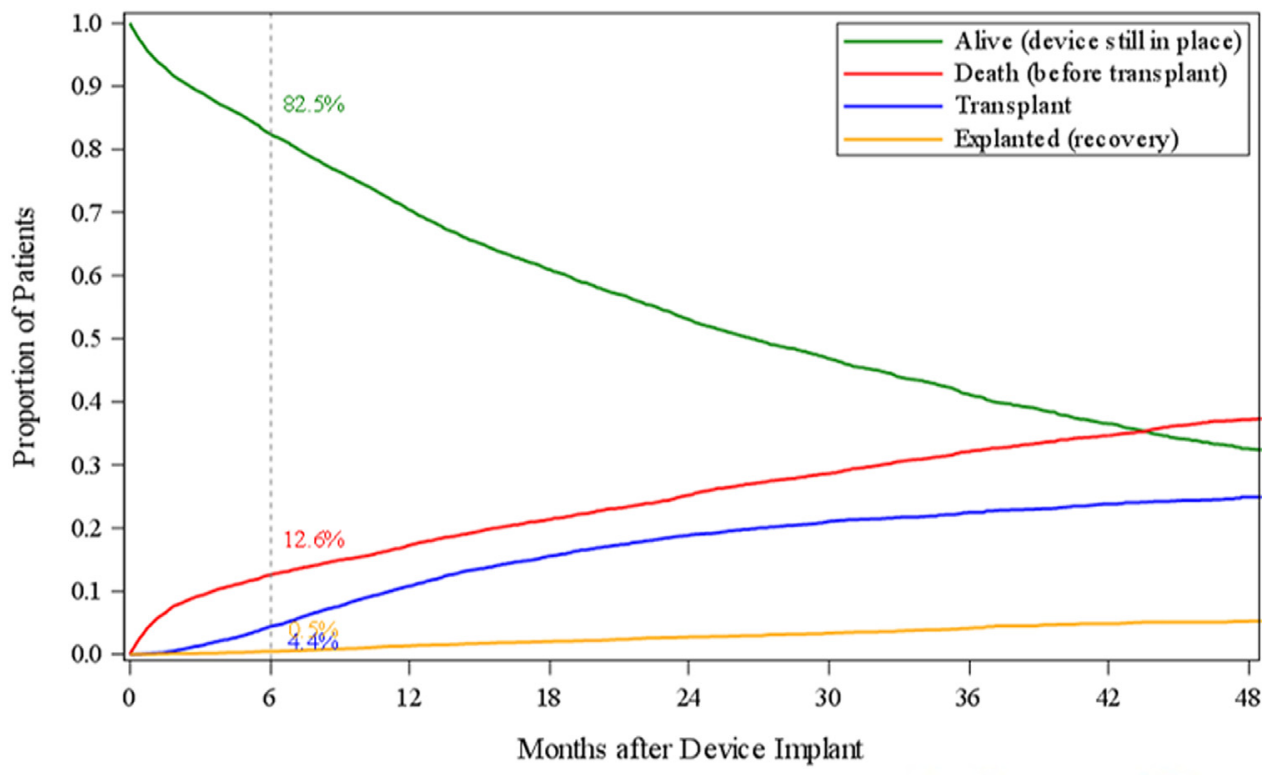

\begin{tabular}{|c|c|c|c|c|}
\hline \multicolumn{5}{|c|}{ Month } \\
\hline $\mathbf{0}$ & $\mathbf{1}$ & $\mathbf{1 2}$ & $\mathbf{2 4}$ & $\mathbf{3 6}$ \\
\hline 9988 & 9382 & 5850 & 3166 & 1452 \\
\hline
\end{tabular}

(b)

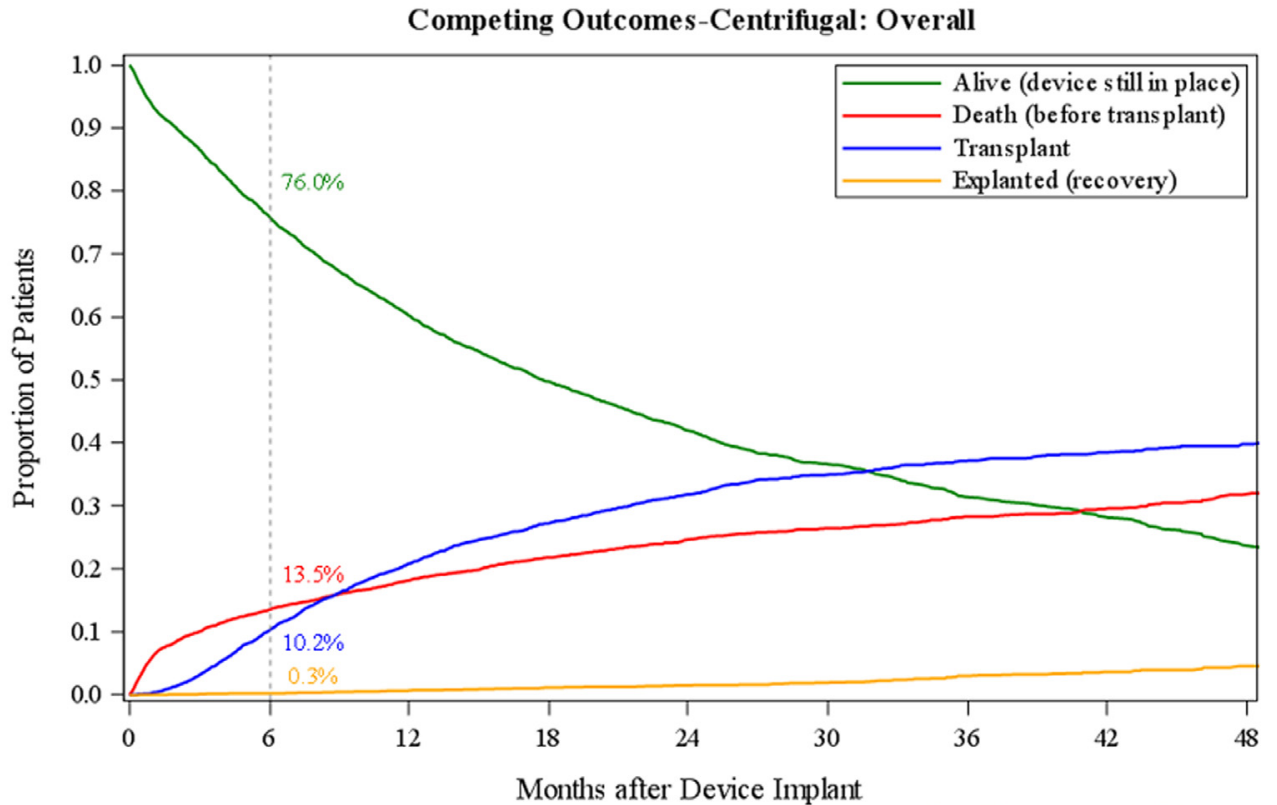

\begin{tabular}{|c|c|c|c|c|}
\hline \multicolumn{5}{|c|}{ Month } \\
\hline $\mathbf{0}$ & $\mathbf{1}$ & $\mathbf{1 2}$ & $\mathbf{2 4}$ & $\mathbf{3 6}$ \\
\hline 5572 & 5145 & 2566 & 1234 & 482 \\
\hline
\end{tabular}

Figure 3 Competing outcomes in IMACS registry according to pump flow. (a) Ax pumps. (b) Centr pumps. Ax, axial flow; Centr, centrifugal flow; IMACS, The International Society for Heart and Lung Transplantation Mechanically Assisted Circulatory Support registry.

Americas and Europe tend to be older than those in the Asia-Pacific region and that destination therapy is the most common implant strategy in the Americas. These demographics, at least partly, explain the differences in survival rates seen in Asia-Pacific region (with younger, bridged-totransplant [BTT] patients with fewer comorbidities).

Because the largest proportion of patients in IMACS derived from the INTERMACS registry (79\%) and most of 
Table 2 Adverse events (AEs) in Sub-Groups: Ax Vs Centr

\begin{tabular}{|c|c|c|c|c|c|c|c|c|c|c|c|c|}
\hline \multirow[b]{2}{*}{ Group } & \multicolumn{2}{|c|}{ GIB } & \multicolumn{2}{|c|}{ D-infection } & \multicolumn{2}{|c|}{$\mathrm{H}-\mathrm{CVA}$} & \multicolumn{2}{|c|}{ I-CVA } & \multicolumn{2}{|c|}{ Thromb } & \multicolumn{2}{|c|}{ RVF } \\
\hline & $A x$ & Centr & $\begin{array}{l}\text { Ax } \\
\text { Tot/Bac/Fun }\end{array}$ & $\begin{array}{l}\text { Centr } \\
\text { Tot/Bac/Fun }\end{array}$ & $A x$ & Centr & $A x$ & Centr & $A x$ & Centr & $A x$ & Centr \\
\hline \multicolumn{13}{|l|}{ Americas } \\
\hline Early $^{\mathrm{a}}$ & 7.86 & 5.24 & $2.11 / 1.76 / 0.08$ & $1.88 / 1.45 / 0.12$ & 0.96 & 1.39 & 1.34 & 1.75 & 1.9 & 0.92 & 0.72 & 1.33 \\
\hline Late $^{b}$ & 2.65 & 1.99 & $1.46 / 1.38 / 0.01$ & $1.56 / 1.45 / 0.01$ & 0.40 & 0.53 & 0.36 & 0.45 & 0.88 & 0.9 & 0.01 & 0.04 \\
\hline \multicolumn{13}{|c|}{ Asia-Pacific } \\
\hline Early $^{\mathrm{a}}$ & 2.23 & 5.22 & $5.74 / 5.60 / 0.14$ & $4.51 / 4.37 / \mathrm{NA}$ & 2.37 & 3.24 & 1.29 & 3.10 & 0.93 & 1.41 & 0.07 & 0.56 \\
\hline Late $^{b}$ & 0.74 & 0.46 & $2.47 / 2.45 / \mathrm{NA}$ & $2.58 / 2.43 / \mathrm{NA}$ & 0.53 & 0.87 & 0.24 & 1.05 & 0.17 & 0.38 & NA & NA \\
\hline \multicolumn{13}{|l|}{ Europe } \\
\hline Early $^{\mathrm{a}}$ & 0.84 & 0.55 & $2.40 / 2.33 / 0.07$ & $1.06 / 0.93 / 0.03$ & 0.15 & 0.14 & 0.44 & 0.49 & 2.25 & 1.31 & 0.73 & 1.17 \\
\hline Late $^{b}$ & 0.07 & 0.87 & $0.89 / 0.76 / 0.01$ & $0.76 / 0.69 / \mathrm{NA}$ & 0.04 & 0.11 & 0.18 & 0.12 & 0.32 & 0.38 & 0.03 & 0.00 \\
\hline \multicolumn{13}{|l|}{ Global } \\
\hline Early $^{\mathrm{a}}$ & 7.27 & 4.29 & $2.31 / 1.98 / 0.09$ & $1.48 / 1.46 / 0.09$ & 0.99 & 1.17 & 1.29 & 1.50 & 2.25 & 1.04 & 0.69 & 1.25 \\
\hline Late $^{\mathrm{b}}$ & 2.39 & 1.41 & $1.80 / 1.40 / 0.01$ & $1.37 / 1.27 / 0.01$ & 0.38 & 0.42 & 0.34 & 0.38 & 0.81 & 0.71 & 0.01 & 0.03 \\
\hline
\end{tabular}

Abbreviations: AE, adverse event; Ax, axial flow; Bac, bacterial infection; Centr, centrifugal flow; D-infection, device-related infection; Fun, fungal infection; GIB, gastrointestinal bleed; H-CVA, hemorrhagic cerebrovascular attack; I-CVA, ischemic cerebrovascular attack; NA, not applicable; RVF, right ventricular failure; Thromb, pump thrombosis; Tot, total number of infection.

All values are prevalence of adverse event presented as rates (i.e., number of events per 100 patient-month).

${ }^{a}$ Early event rate was defined as onset $\leq 3$ months after implantation.

b Late event rate was defined as onset $>3$ months after implantation.

those in INTERMACS are from the US, differences and conclusions must be seen within this context. Recent reports ${ }^{19-24}$ suggest that BMI does not influence patient survival, but it could be speculated that obesity could impact specific AEs. In particular, large body size and associated comorbidities such as diabetes might leave the patient more prone to infection. ${ }^{25}$ Surprisingly, this AE was however less common in the Americas. This could imply that ventricular assist device physicians and staff in the Americas have learned how to manage challenges associated with obesity in LVAD recipients. One multicenter HeartMate II (Ax) study ${ }^{9}$ from Asia-Pacific suggests that body surface area is of importance for survival of $>6$ months and AEs in this sub-group of LVAD recipients, suggesting that regional differences in the management of obese LVAD recipients may exist. Potentially, more information on management strategies for obese patients might improve outcomes in regions outside the Americas, but further studies are required to understand the details of this clinical problem.

Comparison of survival in the different regions is complex and should be performed with caution, taking into consideration organ availability and allocation rules as well as legislative and reimbursement issues concerning destination therapy. Overall, patients from Asia-Pacific had the best short- and long-term survivals. Although destination therapy is not approved for reimbursement in Asia-Pacific, majority of the patients remain alive with the device in place (i.e., not transplanted) at 1 year. When comparing Europe and the Americas, patients on axial support appeared to have better long-term survival, but the numbers were small ( $n=258$ alive after 2 years), and survival differences were not noted between the 2 regions when Centr pumps were compared. The difference in INTERMACS profiles at the time of implantation among regions indicates a greater acceptance to offer devices earlier (i.e., lesser ill patients) in Europe than anywhere else in the world. It is further noteworthy that despite the differences in pre-operative morbidity burden, health care coverage, INTERMACS profiles at the time of implantation, and intensive care unit length of stay during index admission, survival was very similar for LVAD patients in the Americas and Europe. This is interesting especially in light of the marked differences in the use of contemporary devices between the 2 regions (Table 1) and thereby the difference in elective vs acute implantation.

As survival after LVAD implantation has increased dramatically over the past decade, AEs appropriately have been awarded much more attention. In the Americas, the foremost burdening $\mathrm{AE}$ was gastrointestinal bleeding (both in the early and late phases), which was clearly different from the European cohort. Whether practice in the Americas with respect to anti-coagulation and anti-thrombotic management is different is unclear but warrants further study. After the publication of alarming data from the US showing an increase in early-pump thrombosis rates among Ax pump recipients ${ }^{26}$ (which was the foremost burdening $\mathrm{AE}$ in the early phase in Europe), reinforcement of stringent anti-coagulation with international-normalized ratio $2-3$ in addition to aspirin was adopted in most centers worldwide, but uptake of the recommendation may have been higher in the US, which in turn could have led to the higher bleeding rates. Another possible cause of the increased risk of bleeding risk may be demographic differences, especially with respect to age and body size, which may have had implications for this AE. Theoretically, larger patients may have been set at higher pump speeds to support the circulation, which has been associated with gastrointestinal bleeding. ${ }^{27}$ 
(a)

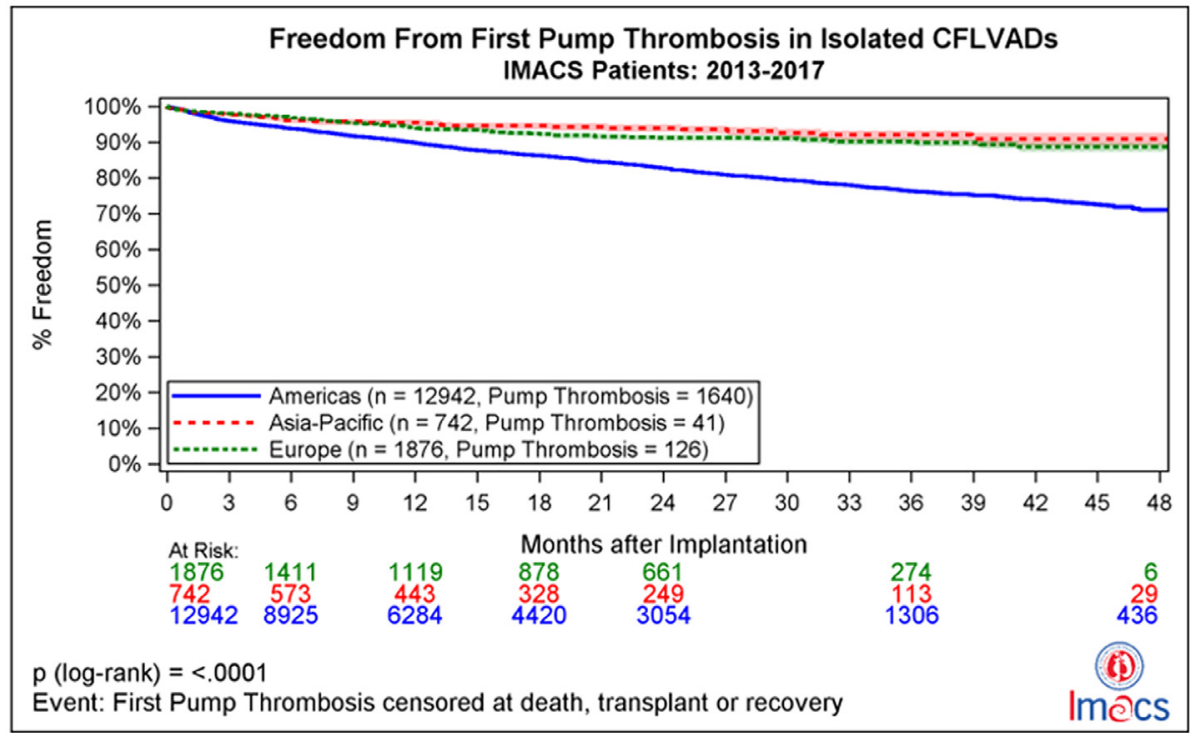

Freedom from first pump thrombosis (in Americas, Asia-Pacific and Europe) $>12$ months was favored in Asia-Pacific.

\begin{tabular}{|l|l|l|l|}
\hline Months after Implant & Americas & Asia-Pacific & Europe \\
\hline 1 & $98.6 \%$ & $98.6 \%$ & $98.7 \%$ \\
\hline 12 & $89.8 \%$ & $95.5 \%$ & $94.0 \%$ \\
\hline 24 & $82.7 \%$ & $94.0 \%$ & $91.2 \%$ \\
\hline 48 & $70.9 \%$ & $90.9 \%$ & $88.6 \%$ \\
\hline
\end{tabular}

(b)

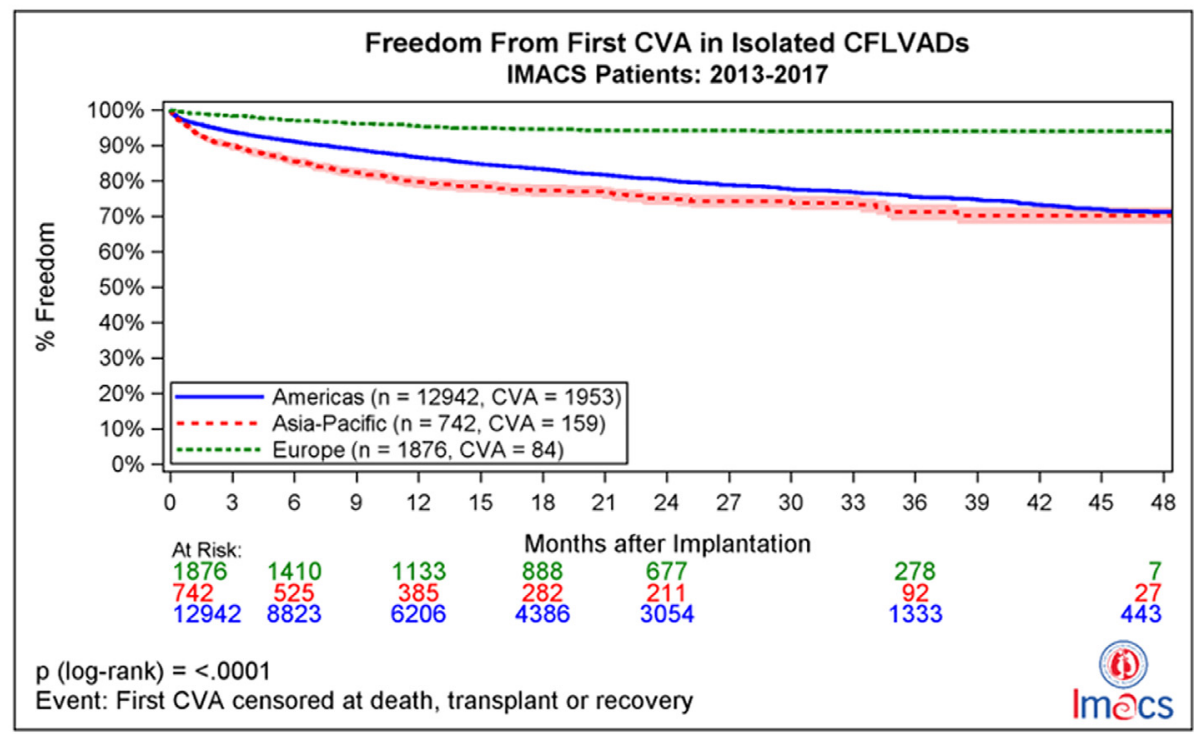

\begin{tabular}{|l|l|l|l|}
\hline Months after Implant & Americas & Asia-Pacific & Europe \\
\hline 1 & $96.5 \%$ & $94.8 \%$ & $99.0 \%$ \\
\hline 12 & $86.6 \%$ & $79.6 \%$ & $95.3 \%$ \\
\hline 24 & $80.2 \%$ & $75.1 \%$ & $94.2 \%$ \\
\hline 48 & $71.2 \%$ & $70.3 \%$ & $94.0 \%$ \\
\hline
\end{tabular}

Figure 4 Regional freedom from first pump thrombosis, CVA, device-specific/related infection, and GI bleeding. (a) Regional freedom from first pump thrombosis in patients implanted with continuous-isolated LVADs in IMACS from 2013 to 2017, $n=15,560$. Freedom from first pump thrombosis in the Americas, Asia-Pacific, and Europe $>12$ months was favored in Asia-Pacific. (b) Regional freedom from CVA in patients with continuous-isolated LVADs in IMACS from 2013 to 2017, $n=15,560$. Freedom from cerebrovascular attack was favored in Europe. (c) Regional freedom from device-specific/related infection in patients with continuous-isolated LVADs in IMACS from 2013 to 2017, $n=15,560$. (d) Regional freedom from GI bleeding in patients with continuous-isolated LVADs in IMACS from 2013 to 2017, $n=15,560$. United Kingdom registry data were excluded from owing to missing data. CFLVAD, continuous-flow LVAD; CVA, cerebrovascular accident; GI: gastrointestinal; IMACS: The International Society for Heart and Lung Transplantation Mechanically Assisted Circulatory Support registry; LVAD, left ventricular assist device. 
(c)

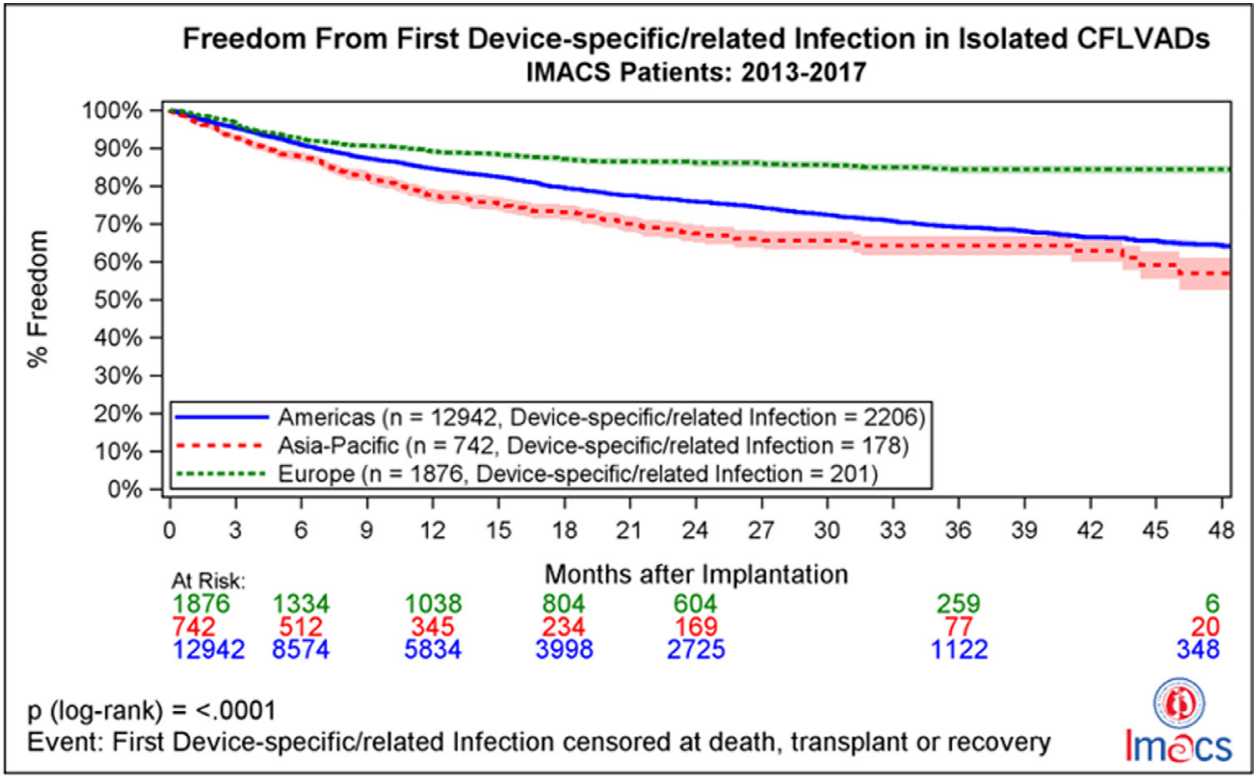

\begin{tabular}{|l|l|l|l|}
\hline Months after Implant & Americas & Asia-Pacific & Europe \\
\hline 1 & $98.4 \%$ & $97.4 \%$ & $99.2 \%$ \\
\hline 12 & $84.6 \%$ & $77.7 \%$ & $89.1 \%$ \\
\hline 24 & $75.9 \%$ & $67.4 \%$ & $86.1 \%$ \\
\hline 48 & $64.3 \%$ & $56.9 \%$ & $84.3 \%$ \\
\hline
\end{tabular}

(d)

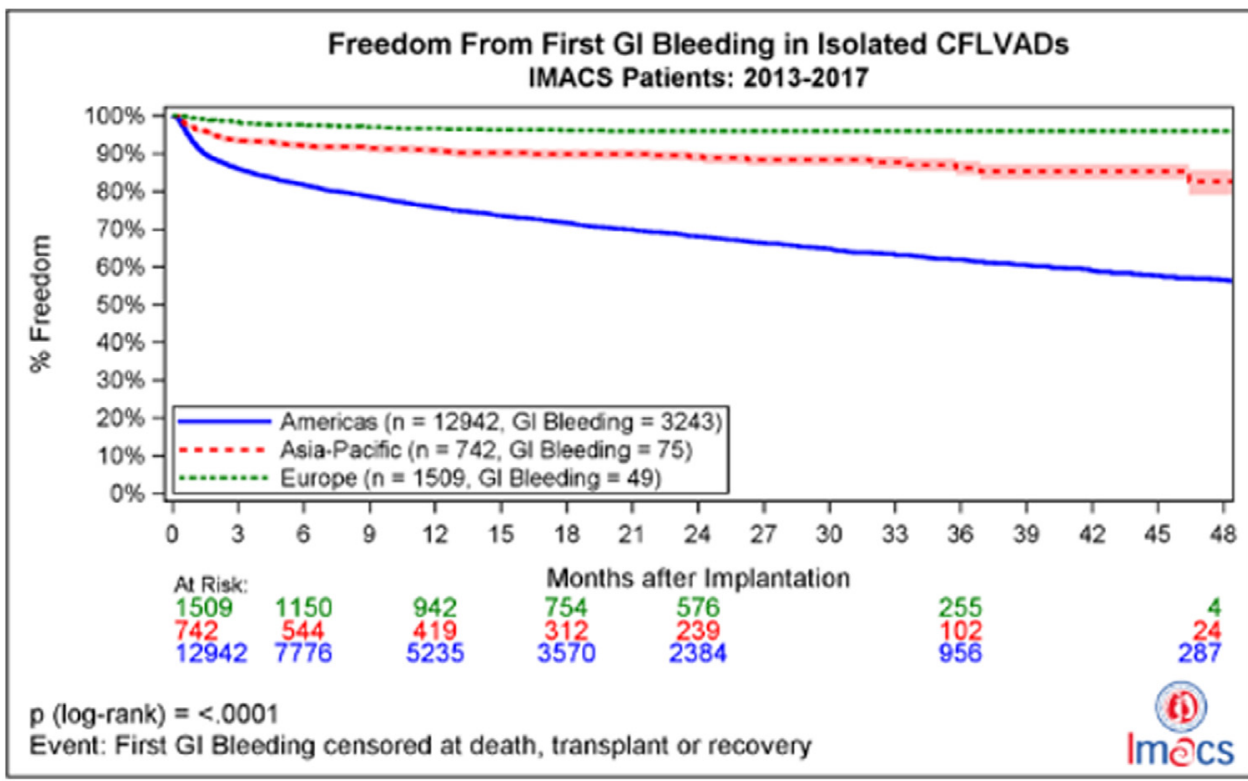

\begin{tabular}{|l|l|l|l|}
\hline Months after Implant & Americas & Asia-Pacific & Europe \\
\hline 1 & $92.7 \%$ & $96.8 \%$ & $99.4 \%$ \\
\hline 12 & $75.7 \%$ & $90.8 \%$ & $96.6 \%$ \\
\hline 24 & $68.0 \%$ & $89.1 \%$ & $95.9 \%$ \\
\hline 48 & $56.5 \%$ & $82.5 \%$ & $95.9 \%$ \\
\hline
\end{tabular}

Figure 4 Continued.

Device-related infection was a leading AE in Asia-Pacific and Europe (except for the early phase of the Centr pump flow device recipients where GIB dominated in AsiaPacific, and in Europe pump thrombosis dominated the early phases of both flow types). Although no specific reasons can be pointed out for the infection rates in this initial analysis, it could represent a regional learning point. Hopefully, this finding might inspire studies evaluating the uptake of recently published recommendations on prophylaxis and management of device-related infections in the different regions and the effect on the burden of this $\mathrm{AE}^{28}$ or even prospective trials to further clarify best practices for 
Table 3 Primary Causes of Death by Region in Recipients of Continuous-Flow LVADs

\section{Americas}

\section{Cause}

Neu

MSOF

RHF

Circulatory

Major infection

\begin{tabular}{ll}
\multicolumn{2}{c}{ Asia-Pacific } \\
\hline Cause & $n(\%)$
\end{tabular}

$638(19)$

$584(18)$

$375(11)$

$243(7)$

218 (7)
Neu

Major infection

MSOF

Major bleeding

Circulatory

$42(45)$
$11(12)$
$9(10)$
$4(4)$
$3(3)$

Europe

\begin{tabular}{llr}
\multicolumn{2}{c}{ Europe } & $p$-value \\
\hline Cause & $n(\%)$ & \\
Major infection & $94(17)$ & $<0.0001$ \\
Neu & $93(17)$ & 0.3162 \\
MSOF & $88(16)$ & 0.0089 \\
RHF & $47(8)$ & 0.3414 \\
Major bleeding & $27(5)$ & 0.1389 \\
\hline
\end{tabular}

Abbreviations: LVAD, left ventricular assist device; MSOF, multisystem organ failure; Neu, neurologic dysfunction; RHF, right heart failure. daily management to provide consistent interregional and intraregional protocols. Whether the variations in the likelihoods of transplantation could be associated with the observed burden of pump thromboses (or if other factors play a larger role) remains to be investigated in future studies. Furthermore, the likelihood of transplantation at 6 months in Asia-Pacific (7.8\%) is clearly explained by the approved device indications (bridge therapy [Australia and Japan]).

It is critical to understand that the findings of this study reflect differential regional patient populations, device availability, and regulatory mandates. In the US, approval for Ax technology as BTT and destination therapy occurred before Centr; thus, a predominance of axial implants is seen in this region. Furthermore, a large number of patients implanted with the newer fully magnetically levitated centrifugal technology as part of an Investigational Device Exemption trial and continued access protocol were not available for inclusion in INTERMACS and thus do not appear in this study. Finally, in Japan, destination therapy is not reimbursed; hence, the use of durable devices is largely limited to a BTT pre-implant strategy.

\section{Limitations and strengths}

This study encompasses several limitations. First, its retrospective design and inconsistent data collection, delays in reporting (leading to bias), differences in reimbursements among regions, differences in follow-up times, and completeness in the different regions (at time of analysis/ writing) should be recognized because these factors not only led to exclusion from analyses but also to limitations in differentiation in neurologic complications (as we were unable to distinguish between cerebral thromboembolic and bleeding events). Second, this study was not designed to describe the rate of tricuspid interventions per se, and the reported rates of this concomitant surgery are not in accordance with prospective, strictly controlled studies (e.g., Multicenter Study of MagLev Technology in Patients Undergoing Mechanical Circulatory Support Therapy With HeartMate 3 trial, conducted in the US, unpublished data). We cannot exclude that the rates of concomitant interventions may have been underreported in the registry. In addition, the reporting in Europe is based on a voluntary registry, which could bias results in this region.
Finally, the results are applicable only to the periods studied during which new devices with other AE profiles were emerging, and as such, extrapolation to the current LVAD landscape should be done with caution.

\section{Conclusion}

Considerable regional variations in patient characteristics, device indications, and outcomes exist. Future studies should explore these differences to identify areas for clinical studies aiming at improving outcomes in LVAD recipients.

\section{Disclosure statement}

K.K.M. received a grant from The Danish Heart Foundation (17R115A742622067). F.G. is a consultant for Abbott and reports having received speaker's fees from Abbott. J.C. is a speaker and consultant for Abbott and Medtronic and further reports being a study advisory board member for Medtronic and Procyrion. D.J.G. is the chair for The International Society for Heart and Lung Transplantation Mechanically Assisted Circulatory Support registry and educator and surgical proctor for Abbott Inc. J.K.K. is the director of The International Society for Heart and Lung Transplantation Mechanically Assisted Circulatory Support Data Coordinating Center (DCC) at University of Alabama at Birmingham and director for Interagency Registry for Mechanically Assisted Circulatory Support DCC. S.M.S. is a consultant for Abbott and reports having received speaker's fees from Abbott. B.M. reports receiving research grants from Abbott. The registry was sponsored by the University of Alabama at Birmingham DCC. The remaining authors have no conflicts of interest to declare.

\section{Supplementary materials}

Supplementary material associated with this article can be found in the online version at https://doi.org/10.1016/j.hea lun.2020.05.001.

\section{Supplementary data}

Supplementary data associated with this article can be found in the online version at www.jhltonline.org/. 


\section{References}

1. Kirklin JK, Cantor R, Mohacsi P, et al. First Annual IMACS report: a global International Society for Heart and Lung Transplantation Registry for Mechanical Circulatory Support. J Heart Lung Transplant 2016:35:407-12.

2. Kirklin JK, Pagani FD, Kormos RL, et al. Eighth annual INTERMACS report: special focus on framing the impact of adverse events J Heart Lung Transplant 2017;36:1080-6.

3. Gustafsson F, Shaw S, Lavee J, et al. Six-month outcomes after treatment of advanced heart failure with a full magnetically levitated continuous flow left ventricular assist device: report from the ELEVATE registry. Eur Heart J 2018;39:3454-60.

4. Gustafsson F, Rogers JG. Left ventricular assist device therapy in advanced heart failure: patient selection and outcomes. Eur J Heart Fail 2017:19:595-602.

5. de By TMMH, Mohacsi P, Gahl B, et al. The European Registry for Patients with Mechanical Circulatory Support (EUROMACS) of the European Association for Cardio-Thoracic Surgery (EACTS): second report. Eur J Cardiothorac Surg 2018;53:309-16.

6. Chatterjee A, Feldmann C, Hanke JS, et al. The momentum of HeartMate 3: a novel active magnetically levitated centrifugal left ventricular assist device (LVAD). J Thorac Dis 2018;10(Suppl 15):S1790-3.

7. Cowger JA, Naka Y, Aaronson KD, et al. Quality of life and functional capacity outcomes in the MOMENTUM 3 trial at 6 months: a call for new metrics for left ventricular assist device patients. J Heart Lung Transplant 2018;37:15-24.

8. Nakatani T, Sase K, Oshiyama H, et al. Japanese registry for mechanically assisted circulatory support: first report. J Heart Lung Transplant 2017:36:1087-96

9. Ono M, Sawa Y, Nakatani T, et al. Japanese multicenter outcomes with the HeartMate II left ventricular assist device in patients with small body surface area. Circ J 2016;80:1931-6.

10. Mehra MR, Uriel N, Naka Y, et al. A fully magnetically levitated left ventricular assist device - final report. N Engl J Med 2019;380:1618-27.

11. Goldstein DJ, Meyns B, Xie R, et al. Third annual report from the ISHLT Mechanically Assisted Circulatory Support Registry: a comparison of centrifugal and axial continuous-flow left ventricular assist devices. J Heart Lung Transplant 2019;38:352-63.

12. Kobashigawa JA, Johnson M, Rogers J, et al. Report from a forum on US heart allocation policy. Am J Transplant 2015;15:55-63.

13. Colvin M, Smith M, Skeans MA, et al. OPTN/SRTR 2015 annual data report: heart. Am J Transplant 2017;17:286-356.

14. Banner NR, Bonser RS, Clark AL, et al. UK guidelines for referral and assessment of adults for heart transplantation. Heart 2011;97:1520-7.

15. Barge-Caballero E, Almenar-Bonet L, Gonzalez-Vilchez F, et al. Clinical outcomes of temporary mechanical circulatory support as a direct bridge to heart transplantation: a nationwide Spanish registry. Eur J Heart Fail 2018;20:178-86.

16. Tsao CI, Chou NK, Chi NH, et al. The influence of the organ allocation policy on a patient's chances of undergoing heart transplantation and the posttransplantation survival rate. Transplant Proc 2012; 44:881-2.

17. Osada K, Imaizumi T, Heart Transplant Candidate Registry Committee of the Japanese Circulation Society. Special report from the heart transplant candidate registry committee in Japan. J Heart Lung Transplant 2005;24:810-4

18. de By TM, Mohacsi P, Gummert J, et al. The European Registry for Patients with Mechanical Circulatory Support (EUROMACS): first annual report. Eur J Cardiothorac Surg 2015;47:770-6. [discussion 776-7]

19. Butler J, Howser R, Portner PM, Pierson RN 3rd. Body mass index and outcomes after left ventricular assist device placement. Ann Thorac Surg 2005;79:66-73.

20. Musci M, Loforte A, Potapov EV, et al. Body mass index and outcome after ventricular assist device placement. Ann Thorac Surg 2008;86:1236-42.

21. Kawabori M, Kurihara C, Sugiura T, et al. Impact of body mass index on implantation of a continuous-flow left ventricular assist device: single-center 508 patient experience. J Am Coll Surg 2017;225(Suppl 1) S30.

22. Yanagida R, Czer LS, Mirocha J, et al. Left ventricular assist device in patients with body mass index greater than 30 as bridge to weight loss and heart transplant candidacy. Transplant Proc 2014;46:3575-9.

23. Go PH, Nemeh HW, Borgi J, Paone G, Morgan JA. Effect of body mass index on outcomes in left ventricular assist device recipients. J Card Surg 2016;31:242-7.

24. Hanke JS, Rojas SV, Avsar M, et al. Impact of body mass index on left ventricular assist device support. J Heart Lung Transplant 2017;36 (Suppl 1):S83.

25. Clerkin KJ, Naka Y, Mancini DM, Colombo PC, Topkara VK. The impact of obesity on patients bridged to transplantation with continuous-flow left ventricular assist devices. JACC Heart Fail 2016;4:7618

26. Starling RC, Moazami N, Silvestry SC, et al. Unexpected abrupt increase in left ventricular assist device thrombosis. N Engl J Med 2014;370:33-40

27. Lopilato AC, Doligalski CT, Caldeira C. Incidence and risk factor analysis for gastrointestinal bleeding and pump thrombosis in left ventricular assist device recipients. Artif Organs 2015;39:939-44.

28. Kusne S, Mooney M, Danziger-Isakov L, et al. An ISHLT consensus document for prevention and management strategies for mechanical circulatory support infection. J Heart Lung Transplant 2017;36:1137-53 